\title{
The Amygdala and Basal Forebrain as a Pathway for Motivationally Guided Attention
}

\author{
Christopher J. Peck ${ }^{1}$ and ${ }^{D}$ C. Daniel Salzman ${ }^{1,2,3,4,5}$ \\ ${ }^{1}$ Department of Neuroscience and ${ }^{2}$ Department of Psychiatry, Columbia University, New York, New York 10027, ${ }^{3}$ Kavli Institute for Brain Sciences, New \\ York, New York 10032, ${ }^{4}$ W.M. Keck Center on Brain Plasticity and Cognition, New York, New York 10032, and ${ }^{5}$ New York State Psychiatric Institute, New \\ York, New York 10032
}

\begin{abstract}
Visual stimuli associated with rewards attract spatial attention. Neurophysiological mechanisms that mediate this process must register both the motivational significance and location of visual stimuli. Recent neurophysiological evidence indicates that the amygdala encodes information about both of these parameters. Furthermore, the firing rate of amygdala neurons predicts the allocation of spatial attention. One neural pathway through which the amygdala might influence attention involves the intimate and bidirectional connections between the amygdala and basal forebrain (BF), a brain area long implicated in attention. Neurons in the rhesus monkey amygdala and $\mathrm{BF}$ were therefore recorded simultaneously while subjects performed a detection task in which the stimulus-reward associations of visual stimuli modulated spatial attention. Neurons in BF were spatially selective for reward-predictive stimuli, much like the amygdala. The onset of reward-predictive signals in each brain area suggested different routes of processing for reward-predictive stimuli appearing in the ipsilateral and contralateral fields. Moreover, neurons in the amygdala, but not BF, tracked trial-to-trial fluctuations in spatial attention. These results suggest that the amygdala and BF could play distinct yet inter-related roles in influencing attention elicited by reward-predictive stimuli.
\end{abstract}

Key words: amygdala; attention; basal forebrain; emotion

\section{Introduction}

Visual stimuli associated with motivationally significant outcomes demand attentional resources. The amygdala is involved in forming associations between stimuli and outcomes (LeDoux, 2000; Baxter and Murray, 2002), and the neurophysiological response properties of individual amygdala neurons reflect these stimulus-outcome associations (Sanghera et al., 1979; SugaseMiyamoto and Richmond, 2005; Paton et al., 2006; Belova et al., 2008; Bermudez and Schultz, 2010). Recent results have shown that the amygdala may also influence spatial attention toward stimuli associated with rewarding outcomes (Vuilleumier et al., 2004; Peck et al., 2013), possibly through amygdala projections to the temporal and occipital cortical areas (Amaral and Price, 1984; Iwai and Yukie, 1987) that in the ventral visual stream (Mishkin et al., 1983). Amygdala projections to the dorsal visual stream, which includes parietal areas known to be important in attention

Received May 23, 2014; revised Aug. 12, 2014; accepted Sept. 2, 2014

Author contributions: C.J.P. and C.D.S. designed research; C.J.P. performed research; C.J.P. analyzed data; C.J.P. and C.D.S. wrote the paper.

This research was supported by grants from the National Institute of Mental Health (NIMH) to C.D.S., as well as by a core grant from the National Eye Institute. C.J.P. received support from NIMH. We thank S. Dashnaw for MRI support, G. Asfaw for veterinary support, and K. Marmon for technical support. We also thank the members of the Salzman Lab for helpful discussions, and Brian Lau for many discussions that aided in the design and analysis of these experiments.

The authors declare no competing financial interests.

Correspondence should be addressed to C. Daniel Salzman, Departments of Neuroscience and Psychiatry, Columbia University, 1051 Riverside Drive, Unit 87, New York, NY. E-mail: cds2005@columbia.edu.

DOI:10.1523/JNEUROSCI.2106-14.2014

Copyright $\odot 2014$ the authors $\quad 0270-6474 / 14 / 3413757-11 \$ 15.00 / 0$
(Corbetta and Shulman, 2002), as well as projections to structures in the frontal lobes that receive input from the dorsal stream, are sparse at best (Amaral and Price, 1984; Barbas and De Olmos, 1990; Baizer et al., 1993). However, the amygdala projects densely to the basal forebrain (BF; Russchen et al., 1985), a collection of subcortical nuclei that are the main source of acetylcholine for the brain (Mesulam et al., 1983); this pathway may be a route through which the amygdala could influence dorsal stream processing.

A number of results indicate that the BF might link the amygdala and dorsal stream attention network. The amygdala and $\mathrm{BF}$ are reciprocally interconnected (Russchen et al., 1985). BF has widespread targets throughout the cortex, including those frontoparietal areas receiving little direct input from the amygdala (Mesulam et al., 1983). Considerable evidence implicates cholinergic projections from BF to cortex in modulating attention (Herrero et al., 2008; Goard and Dan, 2009). BF lesions cause attentional impairments (Voytko et al., 1994; McGaughy et al., 1996) and alter cortical neuron activity normally modulated by attention (Broussard et al., 2009). Finally, connections between the amygdala central nucleus and BF have been implicated in modulating attention (Holland, 2007).

In primates, projections to $\mathrm{BF}$ originate in the amygdala basal and central nuclei (Russchen et al., 1985). Neurophysiological recordings in the basal nucleus of the primate amygdala have revealed that neurons respond selectively to stimuli depending upon their reward associations and spatial location and that these neurons' firing rates are correlated with spatial attention 
allocation (Peck et al., 2013). The neurophysiology of individual primate BF neurons has not been extensively studied, but some results suggest that $\mathrm{BF}$ neurons encode associations between stimuli and motivationally significant outcomes (Richardson and DeLong, 1990, 1991; Wilson and Rolls, 1990). We therefore recorded simultaneously from individual amygdala and BF neurons while reward-predictive stimuli biased spatial attention during performance of a near-threshold visual detection task. Much like amygdala neurons, BF neurons responded selectively depending upon the spatial location and associated reward of stimuli. Amygdala neurons detected the presence of rewardpredicting stimuli appearing in the hemifield contralateral to the recording site more rapidly than BF. Conversely, reward information regarding ipsilateral stimuli appeared earlier in BF. Finally, we observed a trial-by-trial relationship between amygdala firing rates and reaction times, a finding that was not apparent for the BF.

\section{Materials and Methods}

General methods. Two male rhesus monkeys (Macaca mulatta) were used in these experiments, and the general experimental procedures have been described previously (Peck et al., 2013). All experimental procedures complied with NIH guidelines and were approved by the Institutional Animal Care and Use Committees at the New York State Psychiatric Institute and Columbia University.

Task and behavior. Monkeys $\mathrm{O}$ and $\mathrm{D}$ performed a near-threshold detection task designed to assess how monkeys allocate spatial attention depending upon the relationship between previously presented stimuli and reward outcomes (Peck et al., 2013). On each trial, a central fixation point $\left(0.25 \times 0.25^{\circ}\right)$ appeared, and the monkey was required to center its gaze within a window of $2^{\circ}$ around the fixation point. After a fixation period of 500-1500 ms (exponential distribution, $\lambda=170 \mathrm{~ms}$ ), two motivational cues appeared at either side of the fixation point along the horizontal axis ( $7^{\circ}$ eccentricity) for $300 \mathrm{~ms}$. Following the offset of the cues, the monkeys continued to fixate during a delay period in which no peripheral stimuli were present. At a randomly chosen time 400-4000 $\mathrm{ms}$ after motivational cue offset (exponential distribution, $\lambda=390 \mathrm{~ms}$ ), a target appeared for $50 \mathrm{~ms}$ at one of the two locations where the cues had been displayed. Monkeys were required to make a direct saccade to within $3^{\circ}$ of the target between 100 and $600 \mathrm{~ms}$ after its onset and then hold that eye position for $100 \mathrm{~ms}$; successful target acquisition was called a "hit." Liquid reward (when predicted by the cue) was delivered $400 \mathrm{~ms}$ after the hold period; reward consisted of $\sim 1 \mathrm{ml}$ of water controlled by a solenoid and delivered to the monkey through a lick tube. "Miss" trials occurred when the target appeared but monkeys (1) failed to make a saccade (66.6\% of miss trials), (2) made a saccade to the opposite cue location $(23.1 \%$ of miss trials), or (3) made a saccade elsewhere (10.3\% of miss trials).

All trials in which a monkey's eye position left the fixation window before the appearance of the target were repeated such that monkeys were not able to avoid particular trial types; cue configuration, target location, and the length of the delay were re-randomized on repeated trials. Following completed trials (including hit and miss trials), a new trial type was selected at random.

Cues were colored rectangles $\left(2.25 \mathrm{deg}^{2}\right)$, and we randomly interleaved two distinct sets of cues associated with the same outcomes. Targets were Gabor patches; we adjusted the contrast and size of the Gabors on-line to maintain an overall performance level of $\sim 70 \%$ correct. Because the interval during which the target could appear was long and the reaction time window was relatively short, chance performance was $\sim 23 \%$. Maximal chance performance levels were determined by assuming that all saccades were made at the specific time in the trial at which a hit was most likely to "accidently" occur. Given the reaction time window of 100-600 ms, the optimal time to saccade was $100 \mathrm{~ms}$ after the $500 \mathrm{~ms}$ within which targets were most frequent, in which case $\sim 46 \%$ of saccades would occur within the reaction time window. Finally, since these saccades would only be directed at the correct location $50 \%$ of the time, chance performance was determined to be $23 \%$. In practice, saccades times were distributed throughout the trial, suggesting that monkeys did not follow this strategy, and effective chance levels were lower than $23 \%$.

Physiology. Neural recordings were conducted as described previously (Peck et al., 2013). We used MRI and fiducial markers (Brainsight; Rogue Research) to guide placement of the recording chamber and extracellular recording electrodes. Waveforms were collected on-line (Blackrock Microsystems), and single units were isolated off-line using waveform principal components (Plexon Offline Sorter; Plexon). Multi-unit activity (MUA) consisted of waveforms that were not sorted as single units. We re-thresholded the data off-line to correct any major deviations in MUA baseline firing rate due to threshold changes over the course of an experimental session. When MUAs and single-unit activities (SUAs) were recorded on the same channel, we removed MUA time stamps within $2 \mathrm{~ms}$ of any SUA time stamp to ensure that threshold "double-crossings" by the single unit did not contaminate the multi-unit signal. Neurons were recorded primarily in the basolateral nuclei of the amygdala and the nucleus basalis of the BF (Fig. 2B).

In an attempt to classify neurons as either putative excitatory or inhibitory neurons, we used a $k$-means group algorithm ( $n=2$ groups) to group neurons based on their baseline firing rates (last $1000 \mathrm{~ms}$ of the intertrial interval), action potential width (trough to peak), and tendency to fire action potentials bursts as defined in Anderson et al. (2011).

Neural data analysis. For displaying the activity of individual neurons, we computed firing rates in $100 \mathrm{~ms}$ bins slid by $10 \mathrm{~ms}$ (Fig. 2). For all latency analyses, we used firing rates in $30 \mathrm{~ms}$ bins, slid by $1 \mathrm{~ms}$ (Figs. 4-6). Selectivity indices were computed using a receiver-operator characteristic (ROC) analysis to compare firing rate distributions (100-800 $\mathrm{ms}$ after cue onset) between conditions; trials where the target appeared earlier than $800 \mathrm{~ms}$ were truncated at the time of target onset. The significance of firing rate differences across conditions was determined using a Wilcoxon test $(p<0.05)$; highly similar results were obtained by using a bootstrap analysis on selectivity indices. The relationship between selectivity indices was assessed using a linear regression (Fig. 3), and the significance of the slope was evaluated according to the $t$ statistic of the regression $(p<0.05)$.

Latency analysis. For determining latency differences between rewardcontra and reward-ipsi information (Fig. 4), we used the time window $50-400 \mathrm{~ms}$ ( $30 \mathrm{~ms}$ bins, slid by $1 \mathrm{~ms}$ ) after cue onset to determine the significance of selectivity and to find the peak deviation from zero of the firing rate difference for normalization (see below). To compare the latency of population discrimination curves, we continued to use this 50-400 ms time window for one curve while sliding the other in time (always $350 \mathrm{~ms}$ in length) in steps of $1 \mathrm{~ms}$ (see below). For comparing latencies across brain areas (Fig. 5), we used the same 50-400 ms time window for the reward-contra comparison but used a 90-440 ms window for the reward-ipsi comparison given the $\sim 40 \mathrm{~ms}$ lag that we observed in reward latencies (Fig. 4); latencies again were determined by sliding the time window for one curve relative to the fixed time window for the other curve.

We obtained average population discrimination curves across sites by determining the peak deviation from zero of firing rate differences for each site; we then used this value to peak normalize the firing rate differences. This allowed us to average across those neurons with positive and negative selectivity. A cross-correlation was used to determine the optimal shift between population firing rate difference curves, which was defined as the shift that resulted in the minimum squared error between the two curves computed across the entire $350 \mathrm{~ms}$ window; the time windows used for the cross-correlation were the same as those used for determining the significance of selectivity (above). Significance of the shift was determined by bootstrapping where a random set of cells was chosen with replacement on each of 2000 iterations.

We computed visual onset latencies for individual neurons as described previously (Peck et al., 2013). Onset latencies were defined as the first of 15 significant bins (50-500 ms after cue onset compared with 500 $\mathrm{ms}$ before cue onset; $p<0.05$, paired Wilcoxon). This analysis included only visually responsive neurons, which were identified by comparing firing rates $100-300 \mathrm{~ms}$ after cue onset with firing rates recorded during the $500 \mathrm{~ms}$ before cue onset ( $p<0.05$, paired Wilcoxon). 
A

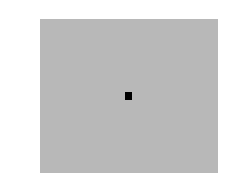

Fix

(500-700 ms)

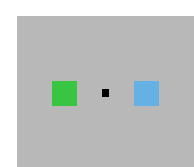

Cues (300 ms)

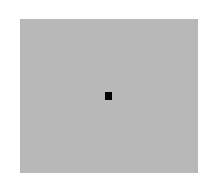

Delay (400-4000 ms)

\section{B}



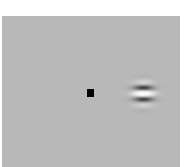

Target (50 ms)
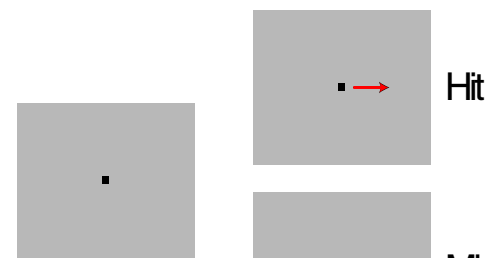

Go (50-550 ms)

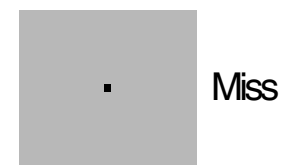

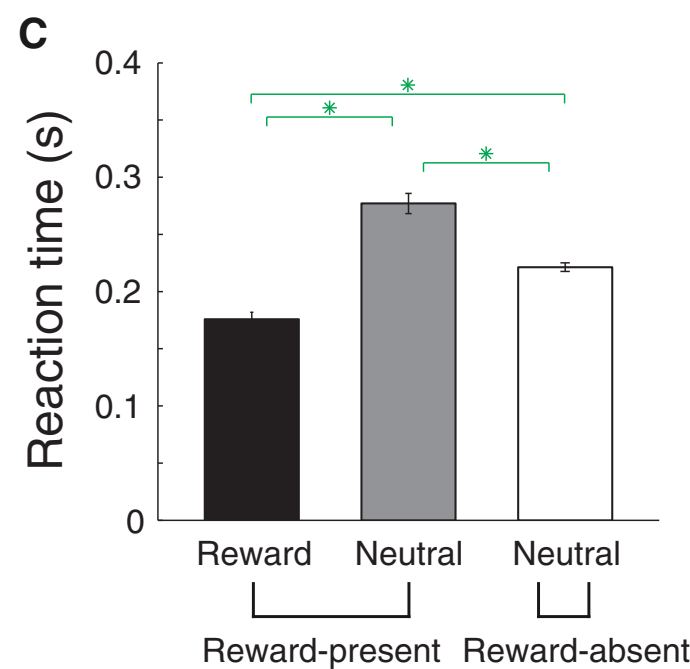

Figure 1. Stimulus-reward associations bias spatial attention. $\boldsymbol{A}$, Detection task. $\boldsymbol{B}$, Percentage correct when the target appeared either at the reward cue location (black) or the neutral cue location on reward-present trials (gray) or on reward-absent trials (white). Green stars indicated significant comparisons (paired Wilcoxon, $p<10^{-8}$ ). C, Reaction time plotted in the same format as $\boldsymbol{B}$.

Reaction time correlation analysis. We calculated correlation coefficients between firing rates $450-800 \mathrm{~ms}$ after cue onset and saccadic reaction times. Before calculating each correlation coefficient, we subtracted the mean firing rate and reaction time for each cue set individually to ensure there were no across-group correlations. After mean subtraction, we calculated the correlation coefficients and applied the Fisher- $Z$ transformation. Correlation coefficients were calculated only if 15 or more trials were available for analysis. We note that the correlation analyses used differ from the approach taken in Peck et al. (2013) (see Fig. 7 of that paper). In the current data, monkeys made a relatively small proportion of saccades to targets appearing at the neutral cue location on reward-present trials (Fig. $1 B$ ). Since there was often no saccade at all on miss trials, we did not have enough data to analyze firing rate by reaction time correlations on these trial types. Thus we could not compute a correlation coefficient difference for the correlations between firing rate and saccadic reaction times for each saccade direction in each condition separately for positive and negative neurons, unlike in Peck et al. (2013), where data from two tasks facilitated the analyses. Data from Peck et al. (2013) analyzed in the same manner as the current paper yielded similar results as those presented here.

\section{Results}

\section{Visual stimuli associated with reward influence} spatial attention

We trained two monkeys to perform a near-threshold visual detection task (Fig. 1A). In this task, visual stimuli associated with different reinforcement outcomes appeared before the visual target that had to be detected (Peck et al., 2013). After establishing fixation, monkeys were presented with two cues appearing on opposite sides of the fixation point. Following a subsequent delay during which the monkey continued to fixate, a target appeared at one of the two spatial locations where the cues had been; the location of the target was chosen randomly and appeared with equal probability at the two spatial locations. The monkey correctly completed the trial (a hit) by making a saccade to the location of the target within $600 \mathrm{~ms}$; miss trials included all those where the monkey failed to saccade to the target.

The two visual cues appearing before the target indicated whether or not monkeys could obtain a reward if the target appeared at that same location; correct performance when a target appeared at the reward cue location resulted in a liquid reward, while correct performance when a target appeared at the neutral cue location resulted in no reward. We interleaved two cue sets to distinguish neural signals encoding stimulus identity from those encoding expected reward. On each trial, either two neutral cues appeared (reward-absent trials) or one neutral and one reward cue appeared (reward-present trials). For reward-present trials, the location of the reward cue was chosen at random and appeared either in the hemifield contralateral or ipsilateral to the brain regions from which we were recording.

We assessed whether monkeys attributed more or less attention to a particular spatial location given the outcome associated with the cue that had appeared there. Of course, cues themselves did not predict where the target would appear, so any influence on measures of spatial attention was due to a cue's reward association, and not the validity of its prediction about target location. We used percentage correct and reaction time to measure attention allocation, both of which are classic measures of spatial attention (Posner et al., 1980). On reward-present trials, when the target appeared at the reward cue location as opposed to at the neutral cue location, percentage correct was higher (Fig. $1 B$; 90 vs $31 \%$; paired Wilcoxon, $p<10^{-10}$ ) and reaction times were shorter (Fig. $1 C$; 176 vs $277 \mathrm{~ms}$; paired Wilcoxon, $p<10^{-10}$ ), indicating that the reward cue biased spatial attention. These 
A

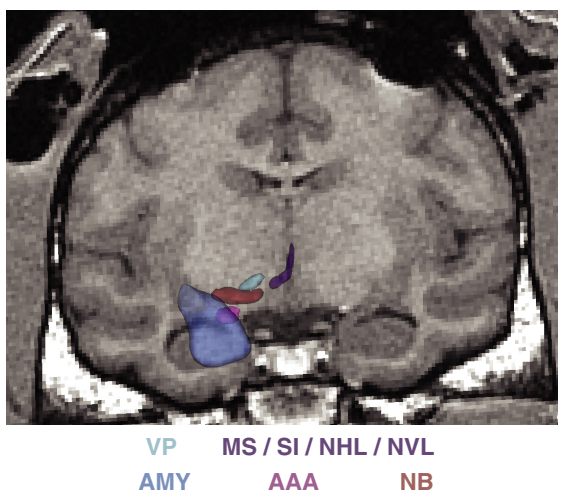

C Amygdala

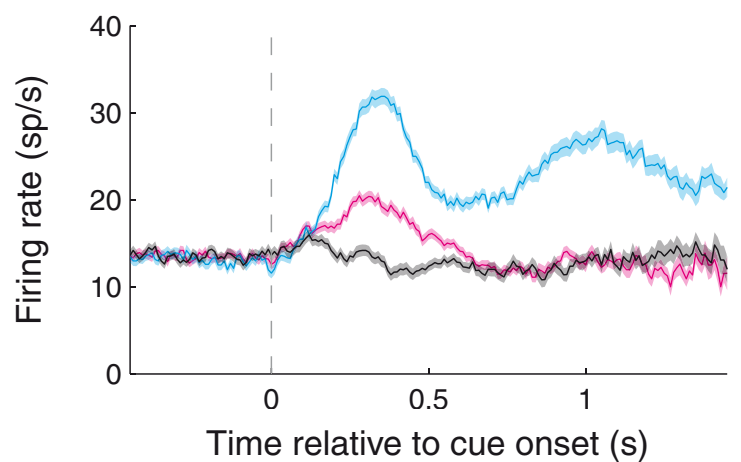

D



B

AMY

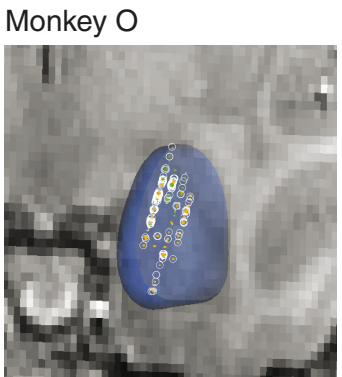

$\mathrm{BF}$

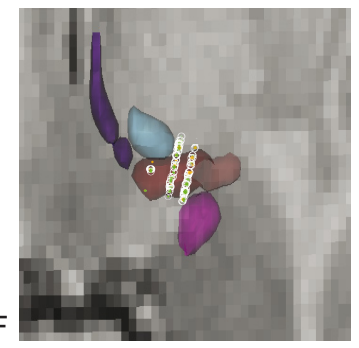

- Reward (+/-) $\bigcirc$ Spatial
Monkey D
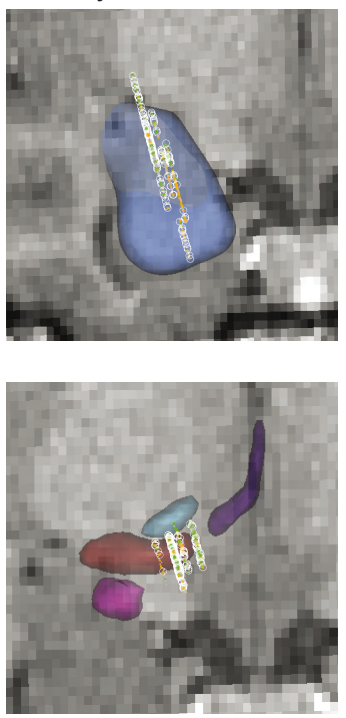

$\odot$ - Reward \& spatial - N.S.
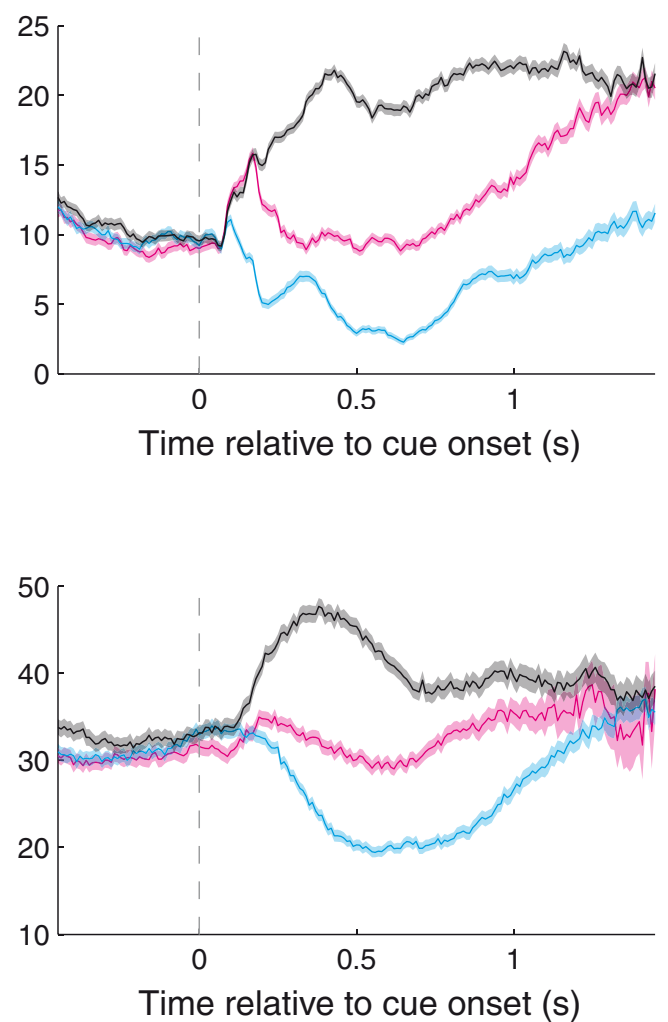

Figure 2. MRI reconstruction of recording sites and individual neuron firing rates. $A$, Sample coronal slice from Monkey $D$ with overlaid $3 D$ reconstructions of the brain areas of interest, as well as nearby brain areas. Light blue: ventral pallidum (VP); purple: medial septal nucleus (MS), substantia innominata (SI), nucleus of the horizontal limb of the diagonal band (NHL), and nucleus of the vertical limb of the diagonal band (NVL); blue: amygdala (AMY); magenta: anterior amygdaloid area (AAA); brown: nucleus basalis (NB). $\boldsymbol{B}$, Recording locations within the amygdala (top) and BF (bottom) for the two monkeys. Plot style indicates the significance of selectivity for individual sites (see legend). C, D, Amygdala and BF neurons respond selectivity to cues depending upon their reward association and spatial configuration. Firing rates as a function of time relative to cue onset for example amygdala $(\boldsymbol{C})$ and BF $(\boldsymbol{D})$ neurons. For each example (SUA) site, both the reward prediction and spatial configuration of the cues had a significant influence on firing rates (Wilcoxon, $p<0.05$; left, reward and spatial selectivity indices $>0.5$; right, reward and spatial selectivity indices $<0.5)$.

effects were true for each monkey considered individually $(p<$ $\left.10^{-3}\right)$. On reward-absent trials, percentage correct and reaction time were intermediate to that in the other two conditions (comparisons with reward-present trials where the target appeared either at the reward or neutral cue location; paired
Wilcoxon, $p<10^{-8}$ ). The performance at the neutral cue location on reward-present trials was therefore likely due to spatial attention being directed away from that location, since performance for the neutral cue was considerably better on reward-absent trials. 


\section{Amygdala and BF neurons are selective for both space and reward}

We recorded SUA and MUA while monkeys performed the detection task for a total of 825 amygdala sites (Fig. 2 A, $B$; 349 SUA, 476 MUA) and 704 BF sites (231 SUA, 473 MUA). We analyzed the data for MUA and SUA together as the results were similar for each; we address this similarity below with respect to the specific analyses. For assessing the magnitude of selectivity for each site, we compared firing rates $100-800 \mathrm{~ms}$ after cue onset across condition by using an ROC analysis to create selectivity indices; the significance of selectivity at each site was determined using an unpaired Wilcoxon test on firing rates $(p<0.05)$.

Neurons in the amygdala (Fig. $2 C$ ) and BF frequently (Fig. $2 D$ ) responded selectively depending upon the reward associated with cues as well as the spatial configuration of the cues. Rewardrelated selectivity was assessed by comparing firing rates on reward-present and reward-absent trials and was significant for a total of $438(53.1 \%)$ amygdala and 424 (60.2\%) BF sites. In each brain area, this consisted of those neurons that fired more on reward-present trials (reward selectivity index $>0.5 ; 219$ amygdala, 268 basal forebrain) and those that fired more on reward-absent trials (selectivity index $<0.5 ; 219$ amygdala, 156 basal forebrain). For spatial selectivity, we compared firing rates on reward-present trials where the reward cue appeared contralateral to the recording site (reward-contra trials) with trials where the rewarded cue appeared ipsilaterally (reward-ipsi trials). Spatial selectivity was prevalent in both brain areas, with 288 (34.9\%) amygdala and 273 (38.8\%) BF sites demonstrating significant spatial selectivity, respectively. Like reward selectivity, the sign of spatial selectivity was mixed across the population; 161 amygdala and $173 \mathrm{BF}$ sites fired more on reward-contra trials (spatial selectivity index $>0.5$ ), and 127 amygdala and $100 \mathrm{BF}$ sites fired more on reward-ipsi trials (selectivity index $<0.5$ ).

One notable difference between the observed selectivity of the amygdala and basal forebrain populations was the proportion of neurons demonstrating positive selectivity (i.e., selectivity indices $>0.5$ ). For reward selectivity, an equal proportion of amygdala neurons exhibited positive and negative selectivity ( 26.5 vs $26.5 \%$ of the entire population), whereas for the basal forebrain, a larger proportion demonstrated positive selectivity (38.1 vs $22.2 \%$ ). The frequency of sites with positive reward selectivity was significantly greater for the basal forebrain $\left(\chi^{2}\right.$ test, $p=0.0001)$. A similar tendency was observed for spatial selectivity; there was a trend $(p=0.0717)$ for reward-contra preferring sites occurring at a higher frequency in the basal forebrain (24.6 vs $14.2 \%$ ) than the amygdala (19.5 vs $15.4 \%)$.

Consistent with our previous results, reward selectivity indices and spatial selectivity indices were positively correlated in the amygdala (Fig. $3 A$; linear regression, $\beta=0.48, p<10^{-66}$ ), indicating that sites with greater firing rates when the reward cue appeared also tended to fire more when that reward cue was contralateral to the recording site. We observed a similar relationship for the basal forebrain (Fig. $3 B ; \beta=0.31, p<10^{-32}$ ). These results were also apparent from the sign agreement of selectivity for individual neurons; of the 183 amygdala and 197 basal forebrain sites exhibiting significant reward and spatial selectivity, the sign of reward and spatial selectivity was the same for 147 and 157, respectively (binomial test, $p<10^{-16}$ for each area). Moreover, the positive correlation between reward and spatial selectivity indices was apparent for each activity type (SUA/ MUA) and each monkey individually ( $p<10^{-6}$ for all comparisons). Overall, these data indicate that the amygdala and BF may coordinate reward and spatial information in a similar manner.
We also determined whether response properties differed between putative excitatory and inhibitory neurons (Fig. $3 C, D$ ). Here, we classified neurons as either putative excitatory (SUA only; 246 amygdala neurons, 154 BF neurons) or inhibitory (34 amygdala, $61 \mathrm{BF}$ ) based on their spike waveforms width, baseline firing rates, and burstiness using a $k$-mean algorithm (see Materials and Methods). There was a significant positive relationship between reward and spatial selectivity indices for each group in each brain area (linear regression, $p<0.001$ ). Further, the slope of these regressions did not differ significantly between the two groups in either brain area (ANCOVA, $p>0.44$ ). We did find, however, differences in the frequency of selectivity among putative excitatory and inhibitory basal forebrain neurons. Putative excitatory BF neurons were more likely to exhibit positive reward selectivity than negative selectivity $\left(77 \%\right.$ vs. $34 \%, \chi^{2}$ test, $p=$ $0.0001)$ and a contralateral preference rather than an ipsilateral preference ( $73 \%$ vs. $41 \%, p=0.0007$ ), but at the same time they were less likely to exhibit significant selectivity in general (reward: $64 \%$ vs. $75 \%, p=0.1166$; spatial: $44 \%$ vs. $72 \%, p=0.0002$ ). On the other hand, putative excitatory and inhibitory neurons in the amygdala did not differ for any of these comparisons $(p>0.14)$. Thus, putative excitatory and inhibitory neurons appear to encode a similar coordination between reward and spatial selectivity, and the excitatory or inhibitory nature of BF neurons is predictive of the sign and existence of selectivity.

Neurons in both brain areas were also selective for stimulus identity, which was determined by comparing firing rates in response to the two distinct stimulus sets. The frequency of sites demonstrating stimulus identity selectivity (17.5\% amygdala, $15.6 \%$ basal forebrain) did not differ across brain area ( $\chi^{2}$ test, $p=0.34)$

\section{Differential latencies for reward and visual information across brain areas}

We examined the latency at which neurons in these areas conveyed reward information to gain insight into the potential sequential processing that occurs between amygdala and BF. Previously, we showed that the latency of reward information in the amygdala depends on the spatial position of stimuli such that neurons detect reward-predicting cues earlier when they appear in the contralateral hemifield (Peck et al., 2013). As a first step in quantifying the latency of reward information, we identified recording sites that exhibited significant selectivity for contralateral and/or ipsilateral reward cues in an early period after cue onset (50-400 ms) by comparing firing rates between reward-contra and reward-absent trials (reward-contra comparison) as well as between reward-ipsi and reward-absent trials (reward-ipsi comparison). For each site and each comparison, we took mean firing rate differences across condition and normalized these firing rate differences (peak normalized and sign corrected, see Materials and Methods) before averaging across the population. We quantify latency differences using a cross-correlation analysis where we held the time window constant for one curve while stepping the other in $1 \mathrm{~ms}$ increments. The optimal time shift was defined as that which resulted in the minimum mean squared error between the two population-level discrimination curves. The rationale for this cross-correlation analysis was to avoid any influence that the strength of signals could have on estimates of latencies when using an analysis method that relies on assessing the statistical significance of a response.

We compared the timing of reward information across cue configuration by examining data from the sites in which firing rates were significantly different in both the reward-contra and 
A

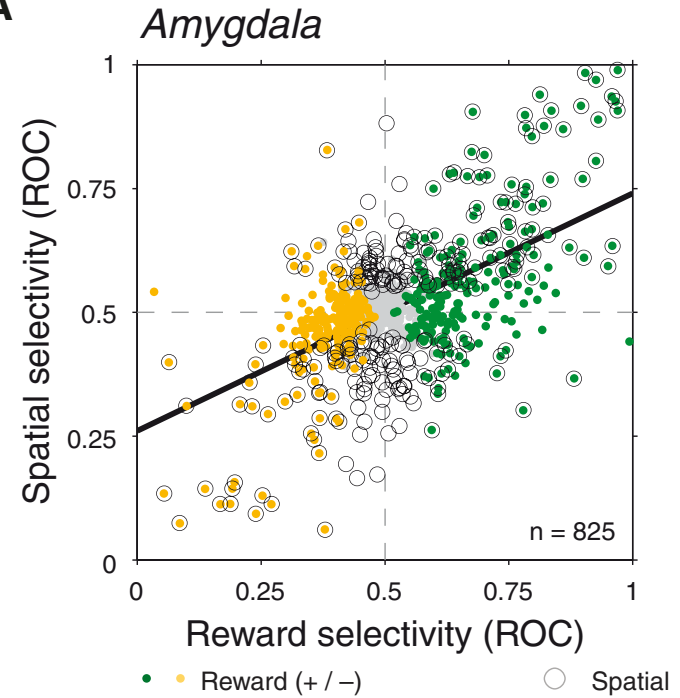

C



E

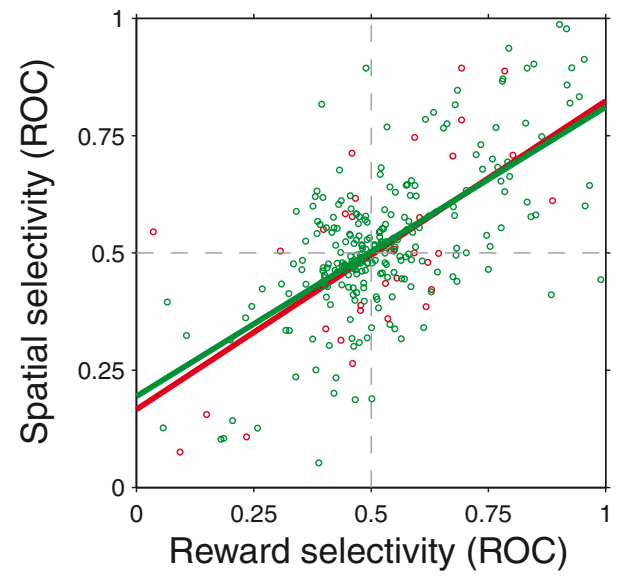

B



- Reward \& spatial

- N.S.

D

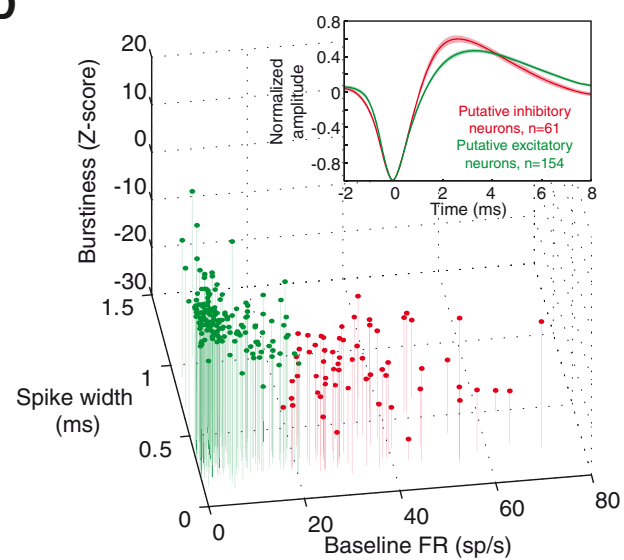

F

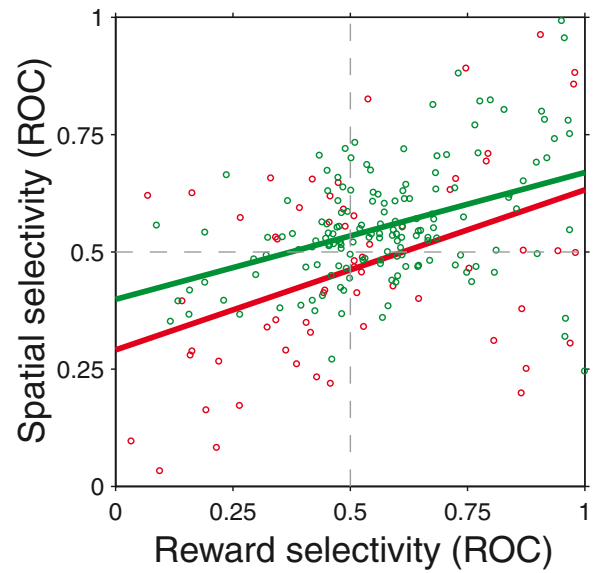

Figure 3. Reward and spatial selectivity are correlated in a similar manner in amygdala and BF and among putative excitatory and inhibitory neurons. $\boldsymbol{A}$, Reward selectivity and spatial selectivity indices are positively correlated for amygdala neurons $\left(p<10^{-66}\right)$. Plotstyle indicates the significance of each (see legend). $\boldsymbol{B}$, Reward selectivity and spatial selectivity indices are positively correlated for BF neurons ( $p<$ $\left.10^{-32}\right) . C, D$, Classification of neurons based on baseline firing rate, spike width, and burstiness. $A k$-means algorithm was used to divide the population of amygdala $(\boldsymbol{C})$ and $B F(\boldsymbol{D})$ neurons into putative excitatory (green) and inhibitory (red) neurons. Insets, Population average waveforms for each group; waveforms were valley normalized before averaging and the shaded region indicates the $S E$ across neurons. $\boldsymbol{E}, \boldsymbol{F}$, Relationship between reward and spatial selectivity as in $\boldsymbol{A}$ and $\boldsymbol{B}$ for putative excitatory (green) and inhibitory (red) neurons. Regressions were significant in each case ( $p<0.001)$.

reward-ipsi comparisons (229 amygdala sites, 296 basal forebrain sites; Wilcoxon, $p<0.05$ ). As expected, we found that contralateral reward information preceded ipsilateral reward information for the amygdala (Fig. 4A; ipsilateral lag of $43 \mathrm{~ms}$; bootstrap, $p<$ $\left.10^{-3}\right)$. We observed the same relationship in the basal forebrain
(Fig. 4B; ipsilateral lag of $34 \mathrm{~ms} ; p<10^{-3}$ ). These results were significant for each activity type (SUA/MUA) and each monkey $\left(p<10^{-3}\right)$.

We next determined whether the timing of reward information differed across brain areas - a result that would shed light on 
A



B

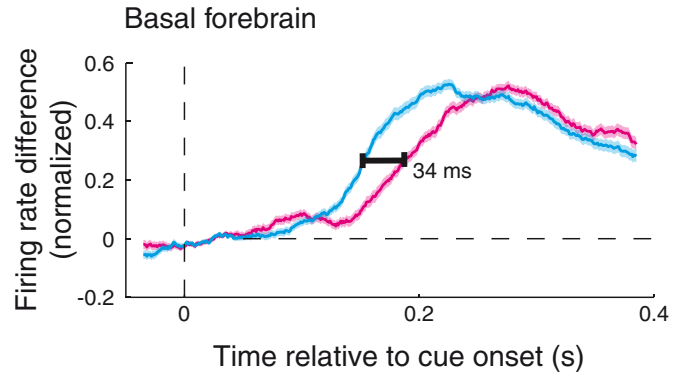

Figure 4. Contralateral reward information appears earlier than ipsilateral reward information in amygdala and BF. A, Population reward-contra (cyan) and reward-ipsi (magenta) discrimination curves as function of time relative to cue onset for amygdala sites. Firing rate differences were peak normalized and sign corrected before averaging over recording sites. The optimal time shift between the curves was determined using data within $50-400 \mathrm{~ms}$ after cue onset; an extended time window is plotted only for display purposes. $\boldsymbol{B}$, Same as $\boldsymbol{A}$ for the BF sites. Latencies differed significantly for each brain area $\left(p<10^{-3}\right)$.

A

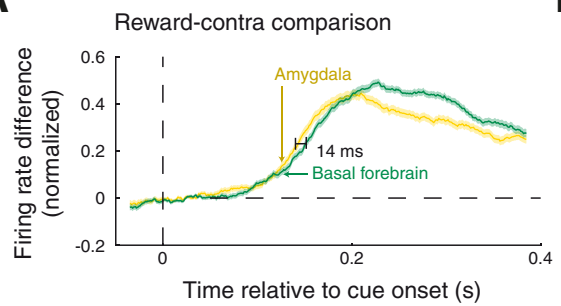

B

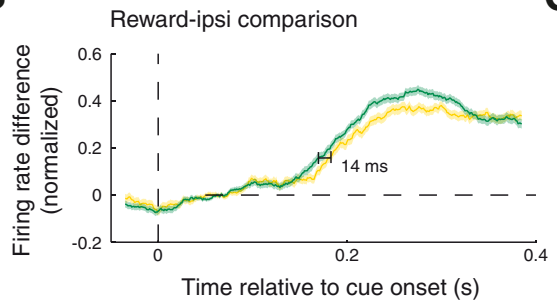

C

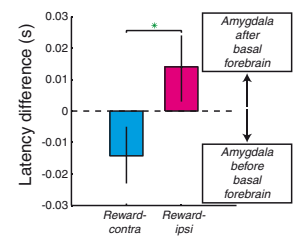

Figure 5. Latency of reward information differs between the amygdala and BF. A, Reward-contra comparison as function of time relative to cue onset for amygdala sites (yellow) and $\mathrm{BF}$ sites (green). Firing rate differences were peak normalized and sign corrected before averaging over recording sites. The optimal time shift between the curves was determined using data within $50-400 \mathrm{~ms}$ after cue onset; an extended time window is plotted only for display purposes. $\boldsymbol{B}$, Same as $\boldsymbol{A}$ for the reward-ipsi comparison. Here, the optimal time shift was determined using data within $90-440 \mathrm{~ms}$ after cue onset. Latency differences were significant in each case $(p<0.05)$. C, Latency differences for contralateral and ipsilateral reward information compared between the amygdala and BF. Error bars indicate the $95 \%$ confidence intervals of the latency differences. Green asterisk indicates the significance of the comparison $(p<0.05)$.

the direction of information flow between amygdala and BF. We compared the latencies across brain areas separately for the reward-contra and reward-ipsi comparisons. Given our finding that ipsilateral reward information appears $\sim 40$ ms later (Fig. 4 ), we adjusted the time windows for analysis to compensate for this lag, using the same 50-400 ms window for the reward-contra comparison as in Figure 4, and a 90-440 ms window for the reward-ipsi comparison. Here, we included all cells that exhibited significant selectivity for a given comparison (reward-contra: 378 amygdala and 423 basal forebrain; reward-ipsi: 316 amygdala and 357 basal forebrain; Wilcoxon, $p<0.05)$. The same time window used for determining selectivity $(50-400 \mathrm{~ms}$ for reward-contra and $90-440 \mathrm{~ms}$ for reward-ipsi) was also used to determine the optimal latency shift between population discrimination curves.

Contralateral reward information appeared earlier in the amygdala than in the basal forebrain (Fig. $5 \mathrm{~A}$; basal forebrain lag of $14 \mathrm{~ms}$; bootstrap, $p=0.002$ ). Surprisingly, this analysis suggested the opposite relationship for ipsilateral reward information, which appeared $14 \mathrm{~ms}$ earlier in the basal forebrain (Fig. 5B; $p=0.014)$. The latency differences themselves were significantly different across trial type (bootstrap, $p<10^{-4}$; Fig. $5 C$ ), which was true for each monkey and activity type $(p<0.05)$. Since these results are based on population data, it is possible that subpopulations of neurons exhibit latencies in disagreement with these population results. Further, anatomical projections between the amygdala and basal forebrain are bidirectional (Russchen et al., 1985), indicating that processing between these two areas is not likely to be purely hierarchical. Nonetheless, these results suggest that, on average, there is a bidirectional flow of reward information between these brain areas where the reward associated with contralateral stimuli is encoded earlier in the amygdala while the reward associated with ipsilateral stimuli is processed first in the basal forebrain.

The earlier latency at which amygdala neurons identify contralateral reward cues (Fig. 5A) may imply that basal forebrain is to some extent dependent on the amygdala for this information. This differential timing could also arise if the brain forebrain depended upon on the amygdala for visual input as well, especially given the lack of direct visual sensory inputs to the basal forebrain (see Discussion). In this case, we would expect to see visual responses in the basal forebrain that were delayed relative to the amygdala. We therefore estimated the visual response latencies of individual neurons and compared the results across brain areas. It was impossible to estimate the visual response latencies of contralateral and ipsilateral stimuli separately (since two cues appeared simultaneously), so we presumed that latencies would be similar for the two stimuli as they would be if the contralateral stimulus was presented alone. For this analysis, we estimated latencies for individual neurons instead of the entire population (Figs. 4,5 ) due to the diversity in the temporal profile of responses that we observed (Fig. 2C,D). Among the population of visually responsive neurons (see Material and Methods), amygdala visual response latencies (mean \pm SE: $108.2 \pm 2.9 \mathrm{~ms}$ ) were significantly shorter than those in the basal forebrain $(120.3 \pm 3.0 \mathrm{~ms}$ ) on reward-contra trials (Fig. 6 A; Wilcoxon, $p<$ $\left.10^{-5}\right)$; this was true individually for each monkey $(p<0.05)$ and for each activity type $(p<0.05)$. Although we focused our analysis on reward-contra trials since these trials were most relevant to the reward-contra comparison (Fig. 5A), amygdala visual responses tended to be earlier on all trial types $(p<0.005$ for 
A

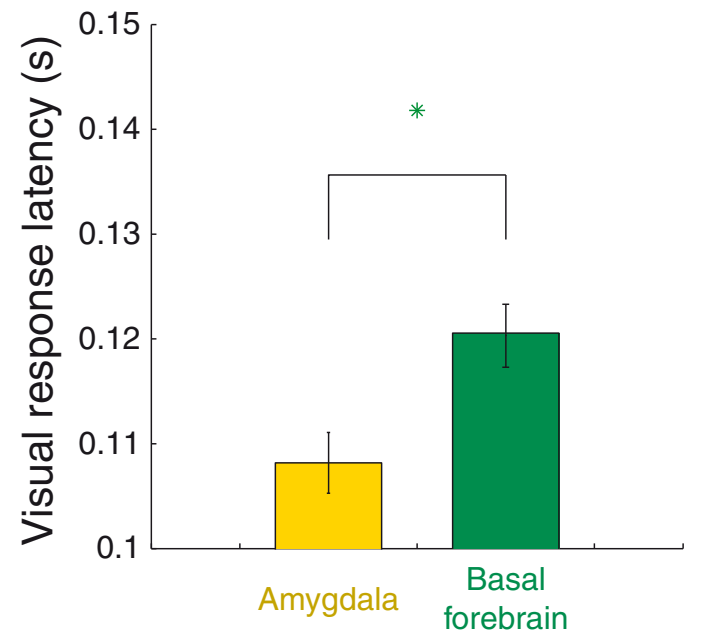

B

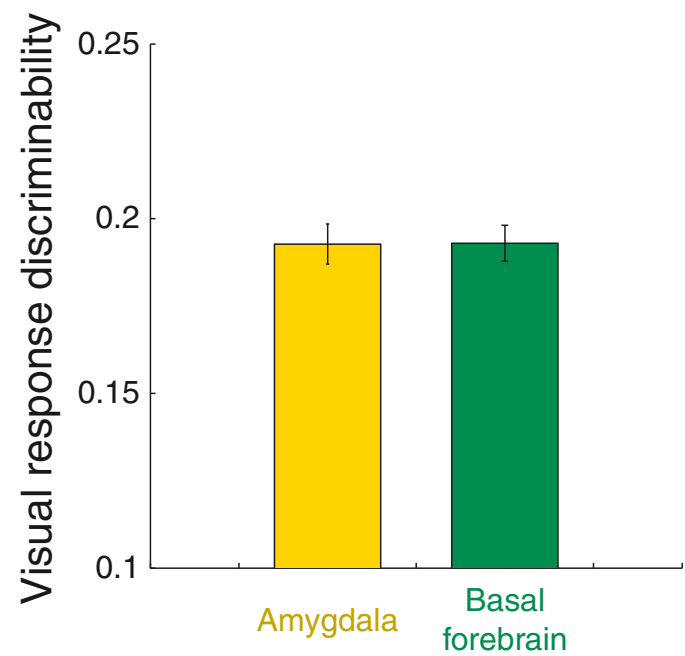

Figure 6. Visual responses occur earlier in the amygdala than the BF. $A$, Mean visual response latencies on reward-contra trials (489 amygdala sites, 523 BF sites). Error bars indicate $S E$ across neurons; green stars denote significant differences across brain area (Wilcoxon, $p<0.05$ ). $\boldsymbol{B}$, Same format and sample as $\boldsymbol{A}$ where the $y$-axis is the absolute R0C value (relative to 0.5 ) comparing baseline firing rates ( $500 \mathrm{~ms}$ before cue onset) with cue-evoked firing rates (100-300 ms after cue onset).

reward-ipsi and reward-absent trials). This latency difference was not driven by a difference in signal strength; the discriminability between baseline and cue firing rates did not differ across brain areas (Fig. 6B; Wilcoxon, $p=0.54$ ).

\section{Amygdala, but not BF, firing rates predict trial-to-trial attention fluctuations}

We previously reported that trial-to-trial variations in spatial attention, as assessed by reaction times, were predicted by amygdala firing rates (Peck et al., 2013). We therefore determined whether basal forebrain neuron firing rates also covary with spatial attention. If basal forebrain neural activity is correlated with spatial attention, then a neuron that responds more strongly when the rewarded cue appears in the contralateral field will tend to fire more on short latency saccades to the contralateral target. In contrast, this neuron would fire less on trials with short latency saccades to the ipsilateral target. This result would be suggestive of a relationship with spatial attention, at least at the hemifield level. On the other hand, if a neuron has a similar relationship between firing rate and reaction times for both saccade directions, it would suggest a nonspatial influence on behavior, akin to arousal or vigilance.

We focused our analysis on those firing rates in the latter half (450-800 ms after cue onset) of the time window used to calculate selectivity indices, given that this time period occurs close to when the target actually appeared. To the extent that the spatial selectivity in the amygdala is tied to the visual stimulus presentation, an analysis of neural activity with respect to the cue onset eliminates any confound related to elapsed time on the strength of the spatial signal, as well as on measures of attention. For each site and each condition, we calculated a correlation coefficient (Fisher $Z$-transformed) between these firing rates and the monkeys' reaction times. Because we expected that the strength and sign of spatial selectivity would be related to the strength and sign of correlations, we first split the data into three equally sized groups according to their spatial selectivity and then split them again based on the sign of spatial selectivity (same indices as in Fig. 3).

We examined whether correlation coefficients differed between saccades directed at the contralateral reward cue location (reward-contra trials) and saccades directed at the ipsilateral reward cue location (reward-ipsi trials). Saccades directed toward the no reward location on these trial types were relatively infrequent (Fig. 1B), so we excluded these conditions. Amongst amygdala sites, correlation coefficients differed significantly between reward-contra and reward-ipsi trials only for the most spatially selective, positive neurons (Fig. $7 A$; $t$ test, $p=0.0008$ ), but not for the most spatially selective negative neurons $(p=$ 0.998). Of note, neither difference was significant when considering a large target-aligned time window for firing rates $(-900$ to $+100 \mathrm{~ms} ; p>0.33$; see Discussion). Correlation coefficients did not differ significantly between saccade directions for any comparison among less spatially selective sites $(p>0.18)$. Overall, those amygdala neurons that fire most when attention is pulled dramatically toward the contralateral hemifield (i.e., on rewardcontra trials) also reflect small variations in attention where firing rate increases correspond to more attention contralaterally and firing rate decreases correspond to more attention ipsilaterally. Unlike the amygdala, correlation coefficients did not differ across saccade direction in any case for the basal forebrain (Fig. $7 B ; p>0.28$ ).

We also analyzed data from reward-absent trials where saccades were more evenly distributed between the contralateral and ipsilateral locations. For amygdala sites, we did not find any differences in correlation coefficients between contralateral and ipsilateral saccades (Fig. $7 C$; $t$ test, $p>0.18$ ). For the basal forebrain (Fig. $7 D)$, there was one instance of a trend ( $p=0.0810$; Fig. $7 D)$, but this appeared to be driven by the data from one monkey (Monkey O: $p=0.5839$; Monkey D: $p=0.0933$ ); all other comparisons for the basal forebrain did not come close to significance $(p>0.57)$.

The primary result that amygdala sites with strong, positive spatial selectivity had firing rates predictive of reaction times was true for each monkey (Fig. $7 E$; Monkey O, $t$ test, $p=0.0308$; Monkey $\mathrm{D}, p=0.0098)$. This result was also significant for SUA $(p=0.0041)$, but appeared only at a trend level for MUA $(p=$ 0.0797); this might have arisen from the fact that subtle trial-totrial variations could be partially washed out when combining data from multiple units. We also repeated this analysis for the 


\section{Amygdala}

A

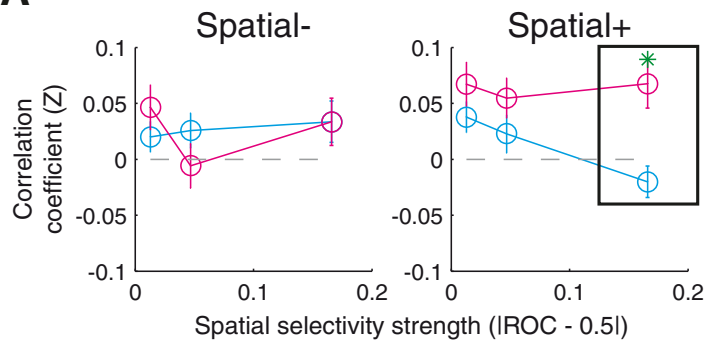

C

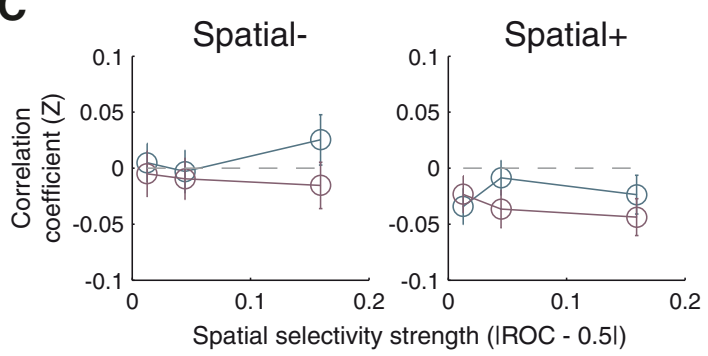

E

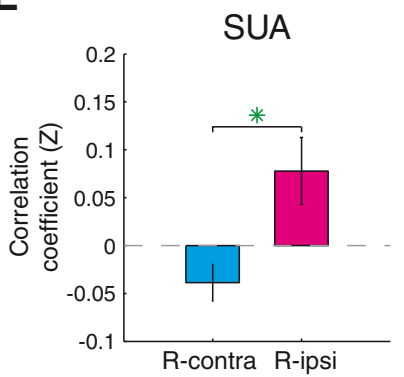

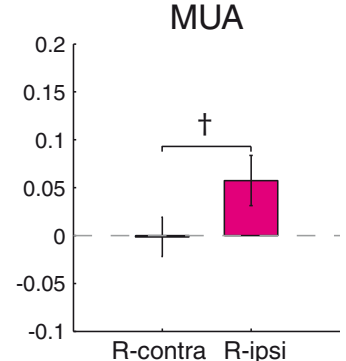

B

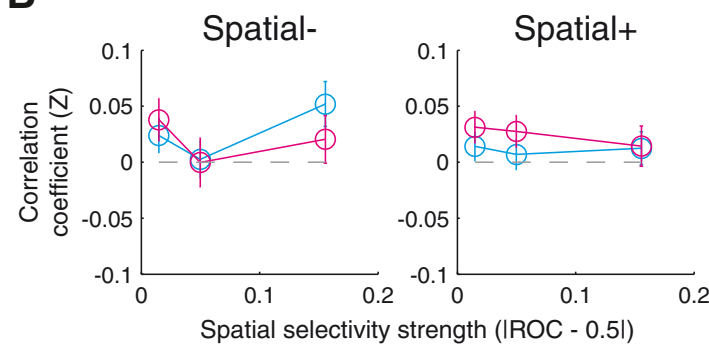

D
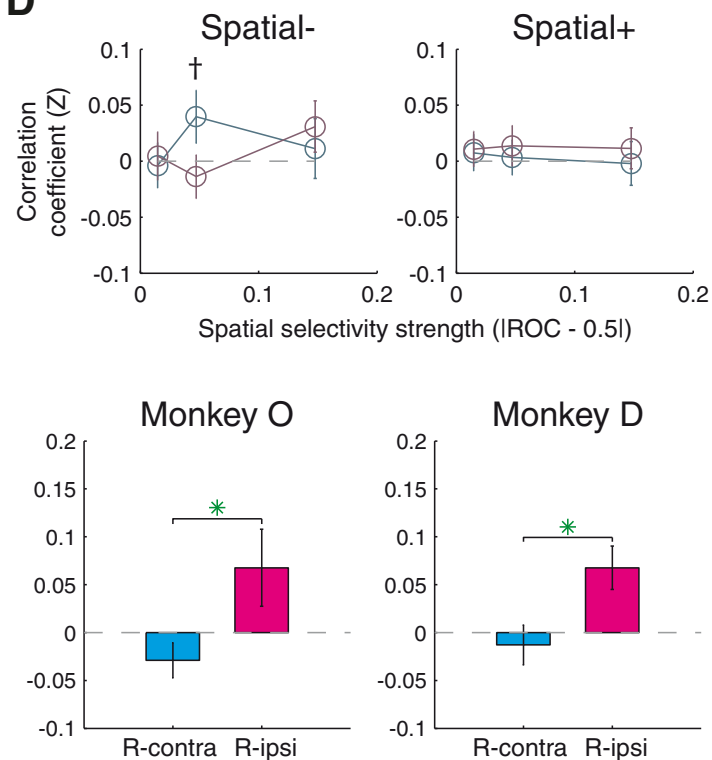

Figure 7. The amygdala, but not the $\mathrm{BF}$, exhibits a trial-by-trial correlation between firing rate and reaction time. $A$, Average correlation coefficients for amygdala sites with negative spatial selectivity (i.e., spatial selectivity index $<0.5$; left) and positive spatial selectivity (i.e., spatial selectivity index $>0.5$; right) for reward-contra (cyan) and reward-ipsi trials (magenta); $x$ values are the mean strength of spatial selectivity (|ROC-0.5|) for each of the three groups. $\boldsymbol{B}$, Same as $\boldsymbol{A}$ for BF sites. $\boldsymbol{C}, \boldsymbol{D}$, Same as $\boldsymbol{A}$ and $\boldsymbol{B}$ except that the data are from reward-absent trials (comparing contralateral and ipsilateral saccades). $\boldsymbol{E}$, Data for the significant comparison (box in $\boldsymbol{A}$ ) split by activity type and monkey. Green asterisks indicate significant comparison ( $t$ test, $p<0.05$ ) and crosses indicate trend effects $(p<0.1)$.

groups of putative excitatory and inhibitory neurons (Fig. 3C,D); a similar effect was present for both groups of amygdala neurons (excitatory: $p=0.0312$; inhibitory: $p=0.0644$ ), and no effect was observed for either group of BF neurons (excitatory: $p=0.6566$; inhibitory: $p=0.9920$ ).

\section{Discussion}

We recorded the activity of individual neurons in the primate amygdala and BF while monkeys allocated spatial attention toward reward-predictive cues. Both amygdala and BF neurons that fired more in response to reward-predictive stimuli also tended to fire more when the reward-predictive cue appeared in the contralateral visual hemifield. Neurons with the opposite reward selectivity tended to have the opposite spatial selectivity (Figs. 2, 3). The latency of reward information pertaining to contralateral stimuli appeared earlier in the amygdala (Fig. 5A), consistent with an amygdala to BF pathway for the processing of rewardpredictive cues appearing contralaterally. Visual responses also had a shorter latency in the amygdala (Fig. 6), suggesting that the $\mathrm{BF}$ might receive both visual and reward information from the amygdala. In contrast, ipsilateral reward information appeared in the BF before the amygdala (Fig. $5 B$ ), possibly indicating distinct neural pathways for conveying contralateral and ipsilateral re- ward information. Amygdala firing rates were correlated with trial-to-trial fluctuations in spatial attention, as measured by saccadic reaction time (Fig. 7). This relationship was not apparent for the BF, suggesting that any influence of BF neural activity on attention might operate on a longer timescale that was not observable in our task.

\section{Interplay of reward information between the amygdala and basal forebrain}

Our observation that the amygdala encodes contralateral reward information before the basal forebrain is consistent with the anatomical pathways that might convey visual and/or reward information to the basal forebrain. Unlike its outputs, which span much of the brain, the basal forebrain receives input from a relatively small subset of brain areas (Mesulam et al., 1983; Mesulam and Mufson, 1984; Ghashghaei and Barbas, 2001). The amygdala is one of a few input sources that might convey information regarding the motivational significance of visual stimuli (Mesulam and Mufson, 1984), making this pathway likely to contribute to enhanced attention to emotionally significant stimuli.

In addition to the amygdala, the prefrontal cortex is a prime candidate for providing reward and/or spatial information to BF. The orbitofrontal cortex (OFC) sends strong projections to the 
basal forebrain (Ghashghaei and Barbas, 2001). OFC neurons are selective for the reward-predictive value of stimuli, but their responses are not strongly influenced by spatial parameters (Wallis and Miller, 2003; Padoa-Schioppa and Assad, 2006). A population spanning the ACC and ventromedial prefrontal cortex (vmPFC) was found to jointly encode spatial and reward information at a relatively short latency ( $\sim 150 \mathrm{~ms}$; Kaping et al., 2011). Inputs from subregions of vmPFC (area 32) to the basal forebrain have been described (Ghashghaei and Barbas, 2001), but it is unclear whether these projection sites overlap with those neurons jointly encoding reward and space. The existence of an ACC (area 24) to basal forebrain projection has not been established (Mesulam and Mufson, 1984).

Conceivably, the basal forebrain could form stimulus-reward associations in parallel with the amygdala, OFC, and other brain areas. This mechanism would require the basal forebrain to receive visual input from pathways where stimulus-reward associations were not already represented. The onset latency of visual signals in the basal forebrain, however, is longer than in the amygdala, suggesting that the basal forebrain may receive visual information from areas such as the amygdala where neurons already signal stimulus-reward associations. There is no evidence for projections from occipital and parietal visual areas to the basal forebrain (Mesulam and Mufson, 1984). Direct visual input may arise from the inferotemporal cortex, but its existence is supported by a single anterograde tracing experiment in which the injection included portions of the perirhinal cortices (Mesulam and Mufson, 1984). These considerations make it unlikely that the basal forebrain receives visual information independently from already formed stimulus-reward associations.

Unlike contralateral reward information, the reward predicted by ipsilateral stimuli was encoded more rapidly in the BF than the amygdala. The route of ipsilateral reward information may involve other brain areas sensitive to reward parameters in the prefrontal cortex (Wallis and Miller, 2003; Padoa-Schioppa and Assad, 2006; Kaping et al., 2011), since the two amygdalae do not project directly to each other (Demeter et al., 1990) and because the hemispheric integration of high-level visual information depends upon cross-hemispheric projections between the prefrontal cortices (Tomita et al., 1999). The earlier latency within $\mathrm{BF}$ for ipsilateral reward information suggests that prefrontal cortices convey this information to BF (Ghashghaei and Barbas, 2001), with the BF projection to the amygdala (Russchen et al., 1985) perhaps accounting for at least some of this delay.

\section{Influence of the amygdala and basal forebrain on attention}

Visuospatial attention can be modulated by both bottom-up and top-down mechanisms (Corbetta et al., 2008). The sudden appearance of a stimulus is known to attract attention and corresponds to a bottom-up process. While there are many ways to induce top-down modulation of attention, we used an approach in which the association between stimuli and reward biased spatial attention (Maunsell, 2004). In principle, the effects of a reward-associated stimulus on attention may occur on different timescales. On one hand, this effect may be relatively rapid in a manner useful for altering the locus of attention from trial to trial. On the other hand, the effects could be longer lasting and influence either spatial or nonspatial attentional processes that extend over the course of more than one trial.

Our results document trial-by-trial correlations between amygdala neural activity and spatial attention, an effect that occurs on a relatively short timescale and is most evident in a time window aligned to cue onset. Of course, it is not clear how long it might take amygdala activity to influence spatial attention, if and when such modulation occurs, but these correlations suggest a possible influence on visuocortical activity and the behavioral allocation of spatial attention. This influence could be realized via several pathways: direct projections to the ventral visual pathway, and indirect pathways through either dopamine neurons in the midbrain (El-Amamy and Holland, 2007) or the BF (Holland, 2007).

The BF pathway could convey reward-related information from the amygdala to brain areas such as the frontal eye fields and lateral intraparietal area, which have a documented role in spatial attention (Bisley and Goldberg, 2003; Moore and Fallah, 2004). Projections from within the nucleus basalis of the BF are organized in an approximately topographic fashion (Mesulam et al., 1983; Ghashghaei and Barbas, 2001), suggesting that the response properties of nucleus basalis neurons may be specialized according to the brain area to which they project. As suggested by the current results, spatially selective BF neurons may have specific effects on frontoparietal networks that mediate attention. In addition, BF neurons may influence spatial processing in either retinotopic, environmental, or peripersonal frameworks, and these types of spatial selectivity could be predictive of their projections to visuospatial areas, the hippocampus (among others), or sensorimotor areas, respectively. Our experiment likely pertains to the retinotopic reference frame, since we used eye movements as a behavioral report. Future experiments should investigate the specificity of BF neural responses to other reference frames in paradigms that use multiple spatial frameworks. Even more ambitiously, it will be important to determine whether a given BF neuron's selectivity predicts the brain area to which it projects.

Extensive literature links BF to attention (Richardson and DeLong, 1991; Voytko et al., 1994; McGaughy et al., 1996), but our data failed to demonstrate a correlation between $\mathrm{BF}$ firing rate and reaction time. This result could be because the action of cholinergic input from BF on cortical synapses may occur on a longer timescale. In this case, a BF influence on attention might be spread across multiple trials in our task, making such a relationship undetectable given our nonblocked design. A substantial population of noncholinergic neurons in the rodent BF has been identified (Gritti et al., 2006), and the activity of putative GABAergic BF neurons has been linked to attentional processes (Lin and Nicolelis, 2008). Although there is little evidence for a substantial noncholinergic population in primate BF, particularly in the nucleus basalis where $90 \%$ of neurons are estimated to be cholinergic (Mesulam et al., 1983), we found that putative excitatory and inhibitory neurons in our sample exhibited similar response properties and neither exhibited a trial-by-trial relationship with attention. These considerations raise the possibility that the functional role of indirect amygdala projections to frontal and parietal cortices, mediated by the BF, have a distinct role from amygdala projections sent to ventral visual areas.

Future studies must understand the relationship between the spatial properties of amygdala and BF neurons and the topography of their cortical projections within a given cortical area. Projections to retinotopically organized brain areas may target neurons that represent specific regions of space. Alternatively, projections may terminate indiscriminately, thereby affecting cortical processing of an entire visual hemifield. The present experiments do not shed light on this circuitry. A detailed understanding of how the amygdala and BF modulate attention will therefore require experiments that more precisely characterize the spatial properties of amygdala and BF neurons, while simultane- 
ously developing approaches for understanding the functional connectivity between these neurons and cortical representations.

\section{References}

Amaral DG, Price JL (1984) Amygdalo-cortical projections in the monkey (Macaca fascicularis). J Comp Neurol 230:465-496. CrossRef Medline

Anderson EB, Mitchell JF, Reynolds JH (2011) Attentional modulation of firing rate varies with burstiness across putative pyramidal neurons in macaque visual area V4. J Neurosci 31:10983-10992. CrossRef Medline

Baizer JS, Desimone R, Ungerleider LG (1993) Comparison of subcortical connections of inferior temporal and posterior parietal cortex in monkeys. Vis Neurosci 10:59-72. CrossRef Medline

Barbas H, De Olmos J (1990) Projections from the amygdala to basoventral and mediodorsal prefrontal regions in the rhesus monkey. J Comp Neurol 300:549-571. CrossRef Medline

Baxter MG, Murray EA (2002) The amygdala and reward. Nat Rev Neurosci 3:563-573. CrossRef Medline

Belova MA, Paton JJ, Salzman CD (2008) Moment-to-moment tracking of state value in the amygdala. J Neurosci 28:10023-10030. CrossRef Medline

Bermudez MA, Schultz W (2010) Responses of amygdala neurons to positive reward-predicting stimuli depend on background reward (contingency) rather than stimulus-reward pairing (contiguity). J Neurophysiol 103:1158-1170. CrossRef Medline

Bisley JW, Goldberg ME (2003) Neuronal activity in the lateral intraparietal area and spatial attention. Science 299:81-86. CrossRef Medline

Broussard JI, Karelina K, Sarter M, Givens B (2009) Cholinergic optimization of cue-evoked parietal activity during challenged attentional performance. Eur J Neurosci 29:1711-1722. CrossRef Medline

Corbetta M, Shulman GL (2002) Control of goal-directed and stimulusdriven attention in the brain. Nat Rev Neurosci 3:201-215. Medline

Corbetta M, Patel G, Shulman GL (2008) The reorienting system of the human brain: from environment to theory of mind. Neuron 58:306-324. CrossRef Medline

Demeter S, Rosene DL, Van Hoesen GW (1990) Fields of origin and pathways of the interhemispheric commissures in the temporal lobe of macaques. J Comp Neurol 302:29-53. CrossRef Medline

El-Amamy H, Holland PC (2007) Dissociable effects of disconnecting amygdala central nucleus from the ventral tegmental area or substantia nigra on learned orienting and incentive motivation. Eur J Neurosci 25: 1557-1567. CrossRef Medline

Ghashghaei HT, Barbas H (2001) Neural interaction between the basal forebrain and functionally distinct prefrontal cortices in the rhesus monkey. Neuroscience 103:593-614. CrossRef Medline

Goard M, Dan Y (2009) Basal forebrain activation enhances cortical coding of natural scenes. Nat Neurosci 12:1444-1449. CrossRef Medline

Gritti I, Henny P, Galloni F, Mainville L, Mariotti M, Jones BE (2006) Stereological estimates of the basal forebrain cell population in the rat, including neurons containing choline acetyltransferase, glutamic acid decarboxylase or phosphate-activated glutaminase and colocalizing vesicular glutamate transporters. Neuroscience 143:1051-1064. CrossRef Medline

Herrero JL, Roberts MJ, Delicato LS, Gieselmann MA, Dayan P, Thiele A (2008) Acetylcholine contributes through muscarinic receptors to attentional modulation in V1. Nature 454:1110-1114. CrossRef Medline

Holland PC (2007) Disconnection of the amygdala central nucleus and the substantia innominata/nucleus basalis magnocellularis disrupts performance in a sustained attention task. Behav Neurosci 121:80-89. CrossRef Medline

Iwai E, Yukie M (1987) Amygdalofugal and amygdalopetal connections with modality-specific visual cortical areas in macaques (Macaca fuscata, M. mulatta, and M. fascicularis). J Comp Neurol 261:362-387. CrossRef Medline

Kaping D, Vinck M, Hutchison RM, Everling S, Womelsdorf T (2011) Specific contributions of ventromedial, anterior cingulate, and lateral pre- frontal cortex for attentional selection and stimulus valuation. PLoS Biol 9:e1001224. CrossRef Medline

LeDoux JE (2000) Emotion circuits in the brain. Annu Rev Neurosci 23: 155-184. CrossRef Medline

Lin SC, Nicolelis MA (2008) Neuronal ensemble bursting in the basal forebrain encodes salience irrespective of valence. Neuron 59:138-149. CrossRef Medline

Maunsell JH (2004) Neuronal representations of cognitive state: reward or attention? Trends Cogn Sci 8:261-265. CrossRef Medline

McGaughy J, Kaiser T, Sarter M (1996) Behavioral vigilance following infusions of 192 IgG-saporin into the basal forebrain: selectivity of the behavioral impairment and relation to cortical AChE-positive fiber density. Behav Neurosci 110:247-265. CrossRef Medline

Mesulam MM, Mufson EJ (1984) Neural inputs into the nucleus basalis of the substantia innominata $(\mathrm{Ch} 4)$ in the rhesus monkey. Brain 107:253274. CrossRef Medline

Mesulam MM, Mufson EJ, Levey AI, Wainer BH (1983) Cholinergic innervation of cortex by the basal forebrain: cytochemistry and cortical connections of the septal area, diagonal band nuclei, nucleus basalis (substantia innominata), and hypothalamus in the rhesus monkey. J Comp Neurol 214:170-197. CrossRef Medline

Mishkin M, Ungerleider LG, Macko K (1983) Object vision and spatial vision: two cortical pathways. Trends Neurosci 6:414-417. CrossRef

Moore T, Fallah M (2004) Microstimulation of the frontal eye field and its effects on covert spatial attention. J Neurophysiol 91:152-162. Medline

Padoa-Schioppa C, Assad JA (2006) Neurons in the orbitofrontal cortex encode economic value. Nature 441:223-226. CrossRef Medline

Paton JJ, Belova MA, Morrison SE, Salzman CD (2006) The primate amygdala represents the positive and negative value of visual stimuli during learning. Nature 439:865-870. CrossRef Medline

Peck CJ, Lau B, Salzman CD (2013) The primate amygdala combines information about space and value. Nat Neurosci 16:340-348. CrossRef Medline

Posner MI, Snyder CR, Davidson BJ (1980) Attention and the detection of signals. J Exp Psychol 109:160-174. CrossRef Medline

Richardson RT, DeLong MR (1990) Context-dependent responses of primate nucleus basalis neurons in a go/no-go task. J Neurosci 10:25282540. Medline

Richardson RT, DeLong MR (1991) Electrophysiological studies of the functions of the nucleus basalis in primates. Adv Exp Med Biol 295:233252. CrossRef Medline

Russchen FT, Amaral DG, Price JL (1985) The afferent connections of the substantia innominata in the monkey, Macaca fascicularis. J Comp Neurol 242:1-27. CrossRef Medline

Sanghera MK, Rolls ET, Roper-Hall A (1979) Visual responses of neurons in the dorsolateral amygdala of the alert monkey. Exp Neurol 63:610-626. CrossRef Medline

Sugase-Miyamoto Y, Richmond BJ (2005) Neuronal signals in the monkey basolateral amygdala during reward schedules. J Neurosci 25:1107111083. CrossRef Medline

Tomita H, Ohbayashi M, Nakahara K, Hasegawa I, Miyashita Y (1999) Topdown signal from prefrontal cortex in executive control of memory retrieval. Nature 401:699-703. CrossRef Medline

Voytko ML, Olton DS, Richardson RT, Gorman LK, Tobin JR, Price DL (1994) Basal forebrain lesions in monkeys disrupt attention but not learning and memory. J Neurosci 14:167-186. Medline

Vuilleumier P, Richardson MP, Armony JL, Driver J, Dolan RJ (2004) Distant influences of amygdala lesion on visual cortical activation during emotional face processing. Nat Neurosci 7:1271-1278. CrossRef Medline

Wallis JD, Miller EK (2003) Neuronal activity in primate dorsolateral and orbital prefrontal cortex during performance of a reward preference task. Eur J Neurosci 18:2069-2081. CrossRef Medline

Wilson FA, Rolls ET (1990) Learning and memory is reflected in the responses of reinforcement-related neurons in the primate basal forebrain. J Neurosci 10:1254-1267. Medline 